\title{
Protective effect of a chronic hypobaric hypoxic environment at high altitude on cardiotoxicity induced by doxorubicin in rats: a $7 \mathrm{~T}$ magnetic resonance study
}

\author{
Yixuan Wan ${ }^{1}$, Dongyong Zhu ${ }^{1}$, Bo $\mathrm{He}^{1}$, Yong Guo ${ }^{2}$, Lei Wang ${ }^{3}$, Duojie Dingda ${ }^{2}$, Angwen Laji ${ }^{4}$, \\ Chunhua Wang ${ }^{5}$, Yonghai Zhang ${ }^{6}$, Fabao Gao ${ }^{1}$
}

${ }^{1}$ Department of Radiology, West China Hospital, Sichuan University, Chengdu, China; ${ }^{2}$ Department of Radiology, Yushu People's Hospital, Qinghai, China; ${ }^{3}$ Molecular Imaging Center, West China Hospital, Sichuan University, Chengdu, China; ${ }^{4}$ Department of Clinical Laboratory, Yushu People's Hospital, Yushu, China; ${ }^{5}$ Sichuan Cancer Hospital and Institute, Sichuan Cancer Center, School of Medicine, University of Electronic Science and Technology of China, Radiation Oncology Key Laboratory of Sichuan Province, Chengdu, China; 'Department of Radiology, The Fifth People's Hospital of Qinghai Province, Xining, China

Contributions: (I) Conception and design: Y Wan, D Zhu, B He; (II) Administrative support: F Gao, Y Guo, D Dingda, A Laji, Y Zhang; (III) Provision of study materials or patients: Y Wan, D Zhu, B He, F Gao; (IV) Collection and assembly of data: Y Wan, D Zhu, B He, L Wang; (V) Data analysis and interpretation: Y Wan, C Wang; (VI) Manuscript writing: All authors; (VII) Final approval of manuscript: All authors.

Correspondence to: Fabao Gao, MD, PhD. Department of Radiology, West China Hospital, Sichuan University, No. 37 Guoxue Road, Chengdu 610041, China. Email: gaofabao@wchscu.cn.

Background: Doxorubicin (DOX)-induced cardiotoxicity (DIC), a major clinical problem, has no effective preventive therapies. We hypothesized that left ventricular (LV) systolic function would be improved in a chronic hypobaric hypoxia environment at high altitude. The purpose of this study was to investigate whether cardiovascular magnetic resonance could reveal the cardioprotective effect of chronic hypobaric hypoxia on DIC.

Methods: In total, 60 rats were randomly assigned to 1 of 6 groups ( $n=10$ per group): the $\mathrm{P}$ group (plain), PD group (plain + DOX), HH group (high altitude), HHD4 group (high altitude + DOX for 4 weeks), HHD8 group (high altitude + DOX for 8 weeks), and HHD12 group (high altitude + DOX for 12 weeks). The rats were transported to either Yushu (altitude: 4,250 m) or Chengdu (altitude: $500 \mathrm{~m}$ ) where they underwent intraperitoneal injection of DOX (5 mg/kg/week for 3 weeks) or saline. Preclinical $7 \mathrm{~T}$ cardiovascular magnetic resonance was performed at weeks 4,8 , and 12 . Tissue tracking was used to measure LV cardiac function and to analyze global and segmental strains. Subsequently, histological and oxidative stress tests were performed to evaluate the protective effect of a high-altitude environment on DIC.

Results: The left ventricular ejection fraction (LVEF) and global and regional strains in the middle, apical, anterior, septal, inferior, and lateral segments (all $\mathrm{P}<0.05$ ) were improved in the HHD4 group compared with the PD group. The global strain was significantly greater in absolute value in the HHD8 and HHD12 groups than in the HHD4 group (all $\mathrm{P}<0.05$ ). Additionally, histological and enzyme-linked immunosorbent assay evaluations supported the in vivo results.

Conclusions: A chronic hypobaric and hypoxic environment at high altitude partially prevented cardiac dysfunction and increased global and regional strain in DIC rat models, thereby minimizing myocardial injury and fibrosis. In addition, by increasing the total duration of chronic hypobaric hypoxia, the global strain was further increased, which was likely due to reduced oxidative stress.

Keywords: Chronic hypobaric hypoxia; strain analysis; doxorubicin-induced cardiotoxicity (DIC); oxidative stress; tissue tracking; magnetic resonance imaging (MRI) 
Submitted Apr 01, 2021. Accepted for publication Aug 06, 2021.

doi: 10.21037/qims-21-360

View this article at: https://dx.doi.org/10.21037/qims-21-360

\section{Introduction}

Anthracyclines are chemotherapy drugs used in the treatment of various cancers, including hematological malignancies, soft tissue tumors, and other malignant tumors. The most representative of these drugs is doxorubicin (DOX) $(1,2)$. However, DOX may cause severe cardiotoxicity that can eventually result in congestive heart failure or death in a dose-dependent and cumulative manner. Nonetheless, DOX is still the first-line clinical treatment for most types of cancer even though the incidence of DOX-induced cardiotoxicity (DIC) can be as high as $30-50 \%$ in breast cancer patients treated with this agent (3). Finding new ways to prevent and reduce DIC during chemotherapy is essential for improving the quality of life of cancer patients.

A chronic hypobaric environment at high altitude, which is similar to ischemic preconditioning and chronic intermittent hypobaric hypoxia, has a significant protective effect on cardiovascular disease $(4,5)$. Recent studies have suggested that ischemic preconditioning confers a cardioprotective effect against DIC in both in vitro cardiomyocytes (6) and in vivo large-animal models (7). Mechanistically, it is now well established that ischemic preconditioning can protect cardiac tissue from oxidative stress injury through enhancement of the endogenous antioxidant defense capacity (8-10). It is likely that the activation of nuclear factor-erythroid 2 related factor $(\mathrm{Nrf2})$ is required for the induction of antioxidative enzymes, which then increase the antioxidant capacity $(11,12)$. Interestingly, compared with other forms of ischemic preconditioning, chronic hypobaric hypoxia at high altitude has a stronger cardioprotective effect due to the adaptive nature of its protective mechanism and improves the tolerance of the heart to acute environments $(13,14)$. However, whether exposure to a chronic hypobaric hypoxic environment at high altitude has a cardioprotective effect on DIC in rats has not been reported.

Cardiac magnetic resonance (CMR) is being increasingly employed to assess myocardial function and deformation by visualizing heart motion in 3D $(15,16)$. Previous studies have demonstrated that myocardial strain can be used to accurately identify subclinical changes and subtle cardiac dysfunction in cardiac myocytes before ejection fraction (EF) reduction (17-19). Strain analysis has been found to be important in the evaluation of global and regional left ventricular (LV) functional impairment (20-22). An increasing number of studies have reported myocardial deformation abnormalities caused by DOX, showing the important diagnostic value of strain analysis in DIC (23-26). However, there are few reports on DIC in a natural high-altitude environment based on CMR measures of myocardial strain.

In this study, we established a rat model of DIC in a natural chronic hypobaric hypoxic environment at high altitude and verified the cardioprotectiveness of this environment with tissue-tracking cardiovascular magnetic resonance imaging (CMR-TT).

\section{Methods}

\section{Study protocol}

All procedures were performed under an approved protocol by the Institutional Animal Care and Use Committee of the Animal Experimental Center, West China Hospital, Sichuan University. The rats were maintained in accordance with the requirements of the National Institutes of Health Guide for the Care and Use of Laboratory Animals and the Association for Assessment and Accreditation of Laboratory Animal Care. All experimental protocols were reviewed and approved by the Experimental Animal Ethics Committee of West China Hospital, Sichuan University, China. Healthy male Sprague-Dawley rats weighing $200 \mathrm{~g}$ were randomly placed into six groups: the $\mathrm{P}$ group (plain group, $\mathrm{n}=10$ ), PD group (plain + DOX group, $n=10$ ), HH group (hypobaric hypoxia group, n=10), HHD4 group (hypobaric hypoxia + DOX for 4 weeks, n=10), HHD8 group (hypobaric hypoxia + DOX for 8 weeks, n=10), and HHD12 group (hypobaric hypoxia + DOX for 12 weeks, $\mathrm{n}=10$ ). The $\mathrm{P}$ and $\mathrm{PD}$ groups were housed for 4 weeks in Chengdu city (altitude, $500 \mathrm{~m}$ ), with the PD group given $5 \mathrm{mg} / \mathrm{kg}$ DOX via intraperitoneal injection on weeks 1, 2, and 3. The HH and HHD4 groups were housed for 4 weeks in Yushu Tibetan Autonomous Prefecture, Qinghai Province (altitude, 4,250 m), while the HHD8 and HHD12 groups were housed in Yushu for 8 and 

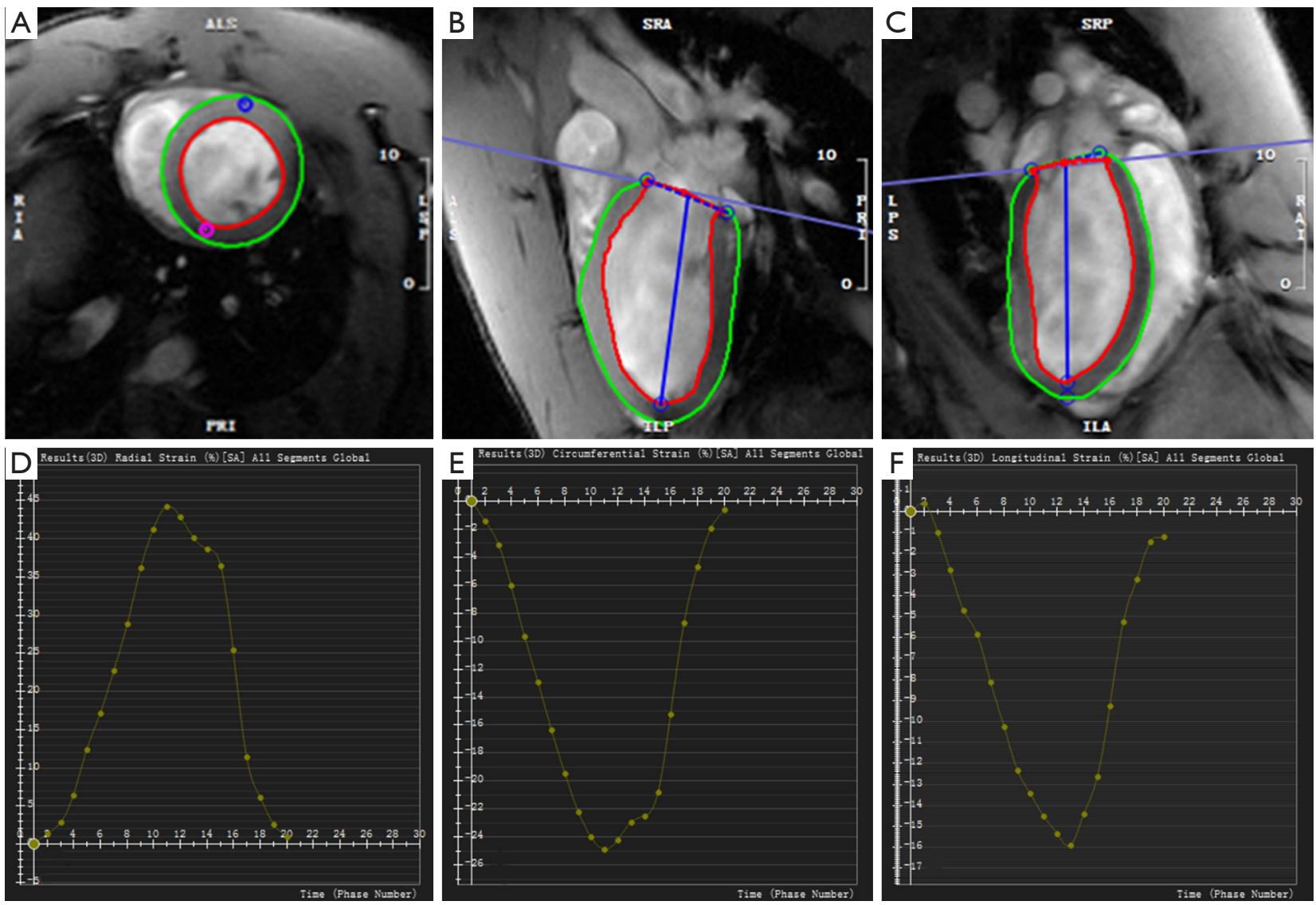

Figure $1 \mathrm{CMR}$ tissue tracking in a healthy control subject using cvi42 software. (A) Cardiac short-axis view; (B) cardiac 2-chamber view; (C) cardiac 4-chamber view; (D) GRS curves; (E) GCS curves; (F) GLS curves (green circle: epicardium; red circle: endocardium). CMR, cardiac magnetic resonance; GRS, global radial strain; GCS, global circumferential strain; GLS, global longitudinal strain.

12 weeks, respectively. The HHD4, HHD8, and HHD12 groups were intraperitoneally injected on weeks 1,2 , and 3 with the same volume of DOX $(5 \mathrm{mg} / \mathrm{kg})$ as was the PD group. The $\mathrm{P}$ and $\mathrm{HH}$ groups received an equal volume of saline solution by intraperitoneal injection on weeks 1,2 , and 3. The rats in the six groups were housed at an animal facility with a temperature of $22 \pm 2{ }^{\circ} \mathrm{C}$ and a 12 -hour lightdark cycle; standard chow and sterile water were available ad libitum.

\section{CMR protocol}

On weeks 4,8 , and 12 , all rats were scanned using a $7 \mathrm{~T}$ magnetic resonance imaging (MRI) system (Bruker BioSpec 70/30, Ettlingen, Germany) with a 4-channel rat surface coil for signal reception. The rats were first anesthetized with isoflurane and then placed prone on the surface coil, with a respiration sensor placed under the abdomen for respiratory gating. Electrodes were placed on 3 of the limbs for electrocardiography (ECG) assessment, and gas anesthesia was maintained with $2 \%$ isoflurane through a pipe connected to a ventilation instrument. IntraGate fast low angle shot (FLASH) MRI, a cardiac cine sequence, was then performed to obtain 2- and 4-chamber views and a stack of short axis images covering the heart from the base to the apex. The following sequence parameters were used: repetition time/echo time (TR/TE), 8.0/2.5 ms; matrix (MTX), 256×256; flip angle (FA), $15^{\circ}$; field of view (FOV), $5 \times 5 \mathrm{~cm}^{2}$; slice thickness, $1.5 \mathrm{~mm}$; and read resolution, $0.26 \times 0.26 \mathrm{~mm} /$ pixel.

\section{Cardiac function and strain analysis}

The CMR images were read using cvi42 software (Circle, version 5.11, Calgary, AB, Canada) (Figure 1). Semiautomatic drawing of the endocardial and epicardial 
borders was performed at the end-diastolic and endsystolic short-axis cine images. The CMR parameters of $\mathrm{LV}$ function were measured, including left ventricular enddiastolic volume (LVEDV), left ventricular end-systolic volume (LVESV), stroke volume (SV), left ventricular ejection fraction (LVEF), and left ventricular mass (LVM).

Myocardial strain parameters of the LV were analyzed. Tissue-tracking (TT) analysis using the same software as above (cvi42 software, Circle) provided LV (I) global longitudinal strain (GLS), global circumferential strain (GCS), and global radial strain (GRS); (II) basal (GLS-b), middle (GLS-m), and apical segment GLS (GLS-a); (III) basal (GCS-b), middle (GCS-m), and apical segment GCS (GCS-a); (IV) basal (GRS-b), middle (GRS-m), and apical segment GRS (GRS-a); and (V) GLS, GCS, and GRS in the anterior, septal, inferior, and lateral LV segments. Endocardial and epicardial borders were drawn on the 2and 4-chamber long-axis cine images and then automatically tracked through all cardiac phases, as shown in Figure 1. All CMR images were analyzed twice by two radiologists.

\section{Oxidative stress assay}

On weeks 4,8 , and 12 , the rats were sacrificed by excessive sodium pentobarbital after CMR. To analyze oxidative stress, rat cardiac tissue was homogenized, and levels of malondialdehyde (MDA), glutathione (GSH), and superoxide dismutase (SOD) activity were measured using assay kits purchased from Nanjing Jiancheng Bioengineering Institute (Jiangsu, China). All procedures were performed according to the manufacturer's protocols.

\section{Histopathological analysis}

A final CMR scan was performed at week 4, after which the hearts were removed by thoracotomy, fixed in $10 \%$ formalin for 24 hours, and cut into 2 -mm-thick slices, which were then embedded in paraffin and cut further into 5 - $\mu \mathrm{m}$-thick sections. Each section was stained with hematoxylin-eosin (HE) and Masson's trichrome (MT) to assess inflammation, necrosis, and fibrosis.

\section{Statistical analysis}

Continuous variables are presented as the mean \pm SD. The Shapiro-Wilk test was used to assess data normality. All data were analyzed by SPSS version 25.0 (IBM Corp., Armonk, NY, USA) and GraphPad Prism 8.0 software
(GraphPad Software Inc., San Diego, CA, USA). Statistical comparisons between groups were assessed by oneway analysis of variance (ANOVA). Correlation analyses between LVEF and global strain and between different global strain forms were performed using the nonparametric Pearson correlation test. Interobserver and intraobserver reproducibility was determined using the intraclass correlation coefficient. $\mathrm{P}<0.05$ was considered statistically significant.

\section{Results}

\section{Basic subject characteristics}

All CMR scans were performed when the heart rate was stable at $390 \pm 20$ beats per minute (bpm) and the respiratory rate was stable at $35 \pm 5 \mathrm{bpm}$. A total of 60 male rats were randomly assigned according to the study protocols. There was no statistically significant difference in baseline characteristics or cardiac function between the $\mathrm{P}, \mathrm{PD}, \mathrm{HH}$, and HHD4 groups (all $\mathrm{P}>0.05$ ) (Table 1).

\section{A high-altitude environment prevented DOX-induced cardiac dysfunction}

CMR analysis revealed that except for LVESV, LV functional and morphological parameters were significantly lower in the PD group than in the P group at week 4. However, after 4 weeks of exposure to a natural hypobaric hypoxic environment at high altitude, there was a statistically significant increase between the HHD4 and PD groups in all LV parameters except for LVEDV and LVESV (Table 2). The results demonstrated that a chronic hypobaric hypoxic environment was effective in alleviating $L V$ cardiac dysfunction after DOX treatment.

\section{A bigh-altitude environment improved $L V$ strain in rats with DIC}

$\mathrm{LV}$ strain analysis revealed that the absolute values of all global and regional strains for the basal, mid-cavity, and apical segments were markedly lower for the PD group than for the $\mathrm{P}$ group. However, compared with the PD group, the absolute values of all global and regional strains for the mid-cavity and apical segments were significantly higher in the HHD4 group but not in the basal segments (Table 3, Figure 2). In addition to the abovementioned segmental strain analysis, we also evaluated LV strain 
Table 1 Baseline characteristics of the study animals

\begin{tabular}{|c|c|c|c|c|c|}
\hline Parameters & $P$ group & PD group & $\mathrm{HH}$ group & HHD4 group & $P$ value \\
\hline HR (bpm) & $323 \pm 22.91$ & $319.4 \pm 29.39$ & $330.8 \pm 35.41$ & $326.4 \pm 22.81$ & 0.83 \\
\hline Breath (bpm) & $40.2 \pm 3.97$ & $37.9 \pm 4.61$ & $38.3 \pm 3.83$ & $39.9 \pm 4.1$ & 0.52 \\
\hline LVEF (\%) & $66.89 \pm 5.75$ & $67.03 \pm 3.6$ & $70.22 \pm 4.52$ & $67.47 \pm 4.74$ & 0.36 \\
\hline LVM (g) & $0.34 \pm 0.03$ & $0.32 \pm 0.02$ & $0.31 \pm 0.03$ & $0.32 \pm 0.03$ & 0.27 \\
\hline LVEDV (mL) & $0.31 \pm 0.05$ & $0.29 \pm 0.03$ & $0.3 \pm 0.04$ & $0.33 \pm 0.07$ & 0.31 \\
\hline LVESV (mL) & $0.1 \pm 0.02$ & $0.09 \pm 0.02$ & $0.1 \pm 0.02$ & $0.1 \pm 0.03$ & 0.12 \\
\hline
\end{tabular}

The results are presented as the mean \pm SD. $\mathrm{P}$ values were obtained using one-way ANOVA. $\mathrm{P}$, plain; PD, plain + doxorubicin; HH, hypobaric hypoxia; HHD4, hypobaric hypoxia + doxorubicin for 4 weeks; HR, heart rate; LVEF, left ventricular ejection fraction; LVSV, left ventricular stroke volume; LVM, left ventricular mass; LVEDV, left ventricular end-diastolic volume; LVESV, left ventricular end-systolic volume.

Table $2 \mathrm{LV}$ functional parameters measured at week 4

\begin{tabular}{|c|c|c|c|c|}
\hline Parameters & P group & PD group & $\mathrm{HH}$ group & HHD4 group \\
\hline LVEF (\%) & $65.82 \pm 4.24$ & $53.26 \pm 7.16^{\star \star \star}$ & $61.86 \pm 3.51$ & $57.64 \pm 3.16^{\#}$ \\
\hline LVSV (mL) & $0.33 \pm 0.04$ & $0.19 \pm 0.04^{\star \star \star}$ & $0.28 \pm 0.03$ & $0.23 \pm 0.05^{\#}$ \\
\hline $\operatorname{LVM}(\mathrm{g})$ & $0.58 \pm 0.08$ & $0.45 \pm 0.07^{\star \star *}$ & $0.54 \pm 0.05$ & $0.46 \pm 0.0^{7 \# \#}$ \\
\hline LVEDV (mL) & $0.5 \pm 0.06$ & $0.36 \pm 0.04^{\star \star \star}$ & $0.46 \pm 0.05$ & $0.37 \pm 0.06$ \\
\hline
\end{tabular}

The results are presented as the mean $\pm \mathrm{SD}$. ${ }^{\star * \star} \mathrm{P}<0.001$ compared with the $\mathrm{P}$ group; ${ }^{\#} \mathrm{P}<0.05,{ }^{\# \#} \mathrm{P}<0.01$ compared with the $\mathrm{PD}$ group. $\mathrm{LV}$, left ventricular; P, plain; PD, plain + doxorubicin; HH, hypobaric hypoxia; HHD4, hypobaric hypoxia + doxorubicin for 4 weeks; LVEF, left ventricular ejection fraction; LVSV, left ventricular stroke volume; LVM, left ventricular mass; LVEDV, left ventricular end-diastolic volume; LVESV, left ventricular end-systolic volume.

using four segments (anterior, septal, inferior, and lateral). Longitudinal, circumferential, and radial strains were significantly decreased in the PD group compared with the $\mathrm{P}$ group in all segments, but a significant increase in four segments was observed in the HHD4 group (Figure 3). These results demonstrated that a chronic hypobaric hypoxic environment was effective in improving $L V$ strain after DOX treatment.

\section{A high-altitude environment alleviated cardiac fibrosis and myocardial injury associated with DIC}

HE staining analysis revealed that the hearts of the PD group had severe infiltration of inflammatory cells, myocardial necrosis, cardiomyocyte vacuolization, and loss of large myocardial fibers. Conversely, cardiomyocyte vacuolization was not present in HHD4 rats, whose myocardial samples showed significantly reduced inflammatory infiltration and conservation of the normal structure of the myocardial tissue (Figure 4A,4B). MT analysis revealed abundant collagen deposition in $\mathrm{PD}$ rats, whereas areas with collagen deposition in HHD4 rats were significantly decreased. There were no significant pathological abnormalities in the $\mathrm{P}$ or $\mathrm{HH}$ groups (Figure $4 C, 4 D)$. In all groups, myocardial lesions were mainly present in the endocardial and midmyocardial layers (Figure $4 A, 4 C)$.

\section{Increasing the total intervention time in a bigh-altitude environment further improved LV global strain}

CMR analysis revealed that the values of LVEF, GLS, GCS, and GRS were significantly lower in the PD group than in the $\mathrm{P}$ group, while after 4 weeks of a high-altitude 
Table $3 \mathrm{LV}$ strain parameters detected at week 4

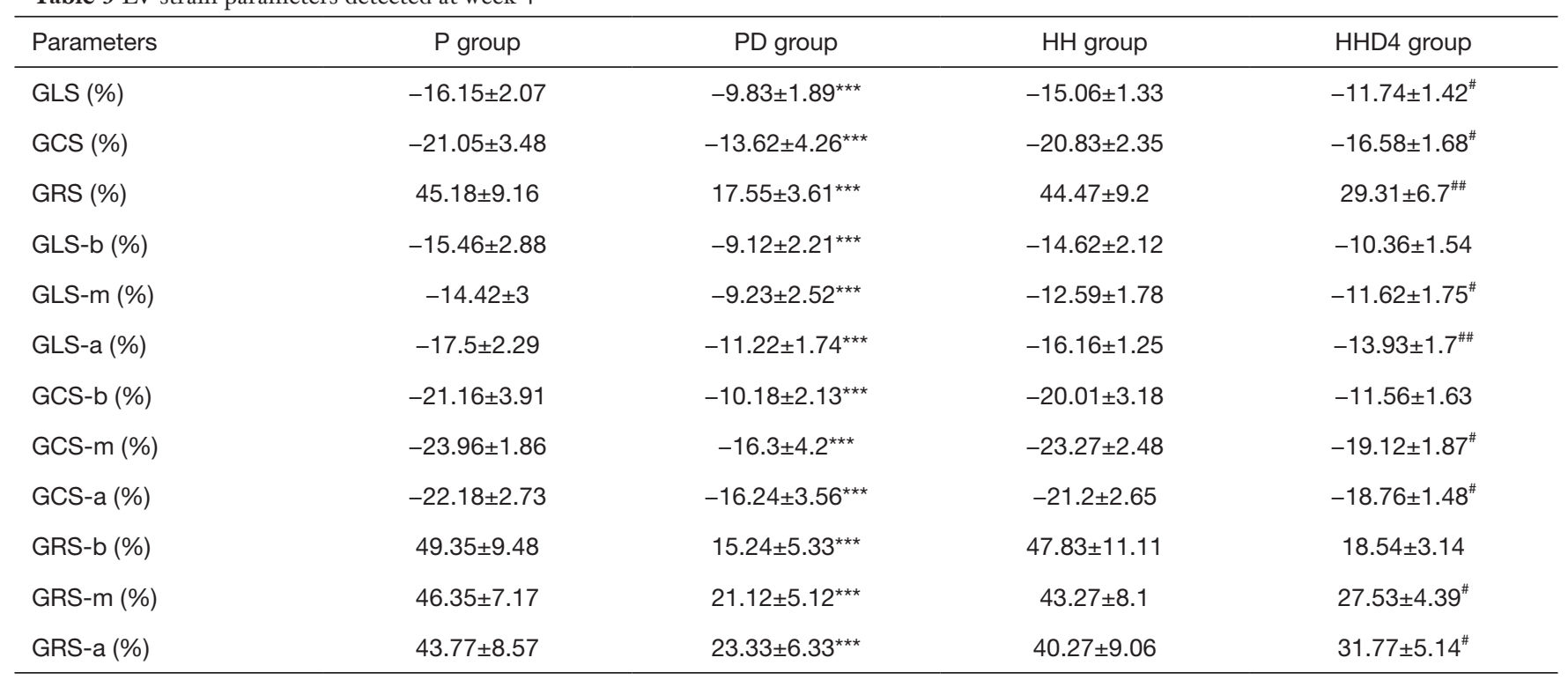

The results are presented as the mean $\pm \mathrm{SD}$. ${ }^{* * *} \mathrm{P}<0.001$ compared with the $\mathrm{P}$ group; ${ }^{\#} \mathrm{P}<0.05$, ${ }^{\# \#} \mathrm{P}<0.01$ compared with the $\mathrm{PD}$ group. LV, left ventricular; P, plain; PD, plain + doxorubicin; HH, hypobaric hypoxia; HHD4, hypobaric hypoxia + doxorubicin for 4 weeks; GLS, global longitudinal strain; GCS, global circumferential strain; GRS, global radial strain; GLS-b, basal segment GLS; GLS-m, mid-segment GLS; GLS-a, apical segment GLS; GCS-b, basal segment GCS; GCS-m, mid-segment GCS; GCS-a, apical segment GCS; GRS-b, basal segment GRS; GRS-m, mid-segment GRS; GRS-a, apical segment GRS.

environment (HHD4), these parameters were increased. In addition, we found that increasing the total exposure time to a high altitude (from 8 to 12 weeks) further increased the absolute value of global myocardial strain but did not affect LVEF. No obvious differences in LVEF, GLS, GCS, or GRS were observed between the HHD8 and HHD12 groups (Table 4).

\section{Increasing the total intervention time in a high-altitude environment reduced oxidative stress in the heart}

To investigate the underlying reason for the further rise in global myocardial strain after increasing the total exposure time to a high altitude, we measured oxidative stress markers in hearts from the five groups. MDA levels were significantly increased following DOX administration (PD group) (Figure $5 A$ ), while these levels significantly decreased after 4,8 , and 12 weeks at high altitude (the HHD4, HHD8, and HHD12 groups, respectively). In contrast, GSH and SOD activity decreased in the PD group (Figure 5B,5C) but increased significantly in the HHD4 group and further increased in the HHD8 and HHD12 groups. No obvious differences in MDA, SOD, or GSH activity were observed between the HHD8 and HHD12 groups (Figure 5A-5C).

\section{Relationship between LVEF, LV strain, and histological fibrosis}

The intra- and interobserver reliability of the measurement of LVEF and LV strain parameters is summarized in Table 5 . Correlation analysis showed that LVEF was significantly negatively correlated with GLS and GCS but significantly positively correlated with GRS (Figure $6 A-6 C$ ). Moreover, there was a significant positive correlation between GLS and GCS and a significant negative correlation of GRS with GLS and GCS (Figure 6D-6F). Finally, GLS and GCS correlated positively with the percentage of fibrosis in the histology, while a negative correlation was found between GRS and histological fibrosis (Figure 6G-6I).

\section{Discussion}

We assessed the cardioprotective effect of a high-altitude environment in a rat model of DIC. CMR evaluation showed that a high-altitude environment significantly attenuated cardiac dysfunction associated with DIC. Strain analysis showed that hearts treated with DOX and exposed to a high-altitude environment had significantly higher global and regional myocardial strains in all segments, 


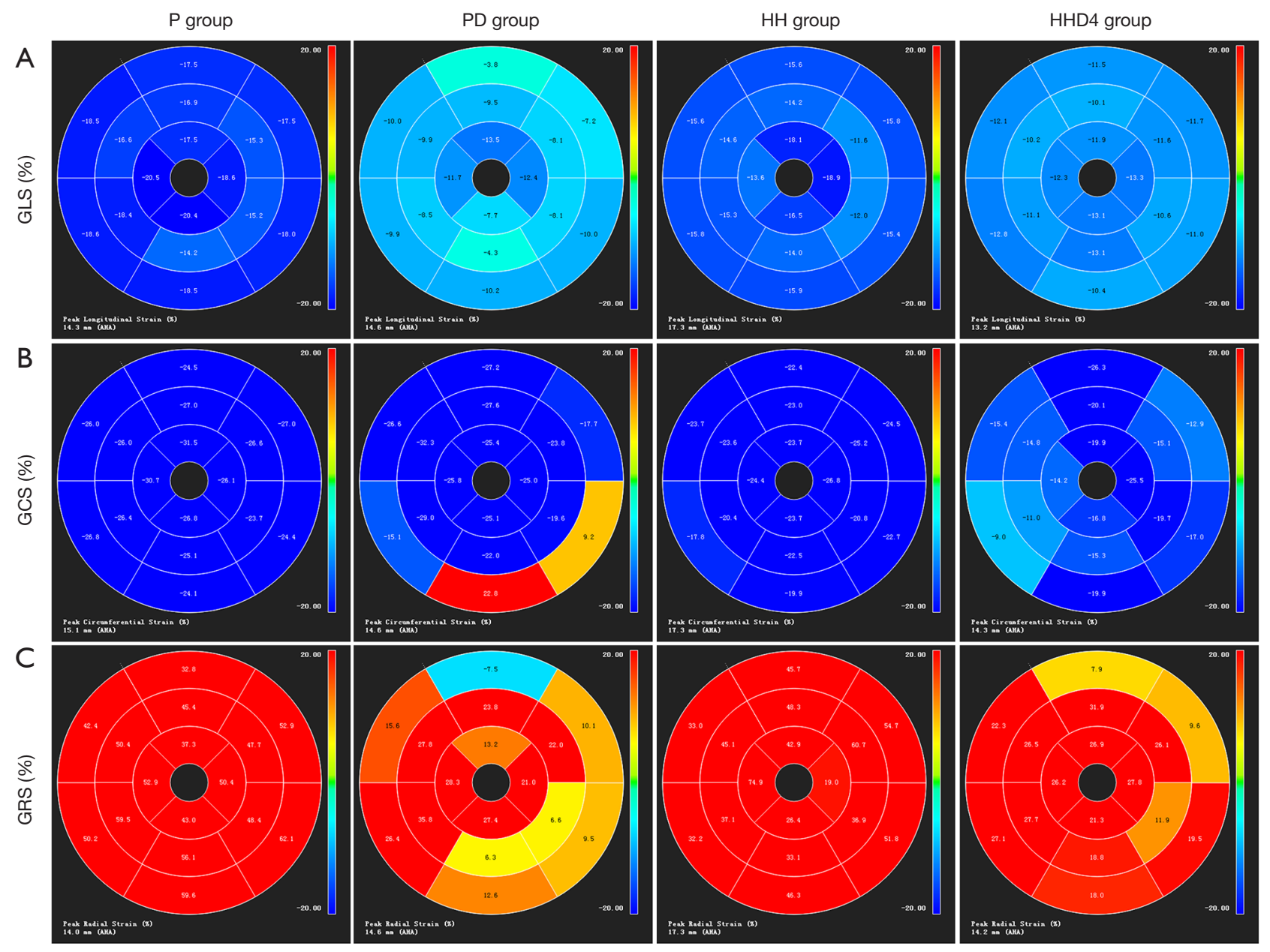

Figure 2 Representative 16-segment myocardial strain imaging in the four groups by CMR. (A) Example of 16-segment GLS. (B) Example of 16-segment GCS. (C) Example of 16-segment GRS. CMR, cardiac magnetic resonance; GLS, global longitudinal strain; GCS, global circumferential strain; GRS, global radial strain; P, plain; PD, plain + doxorubicin; HH, hypobaric hypoxia; HHD4, hypobaric hypoxia + doxorubicin for 4 weeks.

except for the basal segments. Furthermore, we found that increasing the total exposure time to a high-altitude environment for 8 and 12 weeks further increased $\mathrm{LV}$ global myocardial strain but had no effect on the LVEF. In addition, HE and MT staining showed that a chronic hypobaric hypoxic environment at high altitude had a cardioprotective effect on DIC. Finally, we revealed that the protective effect of the total intervention time in a chronic hypobaric hypoxic environment at high altitude on DIC was closely related to oxidative stress.

DOX is one of the most highly effective and frequently used anticancer drugs; however, prolonged use of DOX can cause serious dose-dependent cardiotoxicity, limiting its clinical application (27). Although numerous studies have focused on reducing DIC or seeking alternatives to DOX, DOX remains the primary clinical antitumor agent and to date, there are no effective preventive or therapeutic modalities to reduce DIC. Therefore, there is an urgent need to find a strategy to prevent and treat this condition.

Previous studies have demonstrated the protective effect of high altitude on mortality from coronary heart disease and stroke (28). In an in vivo model of humans, Tibetan patients with congenital heart disease exhibited increased resiliency to ischemia-reperfusion (I/R) injury during cardiac surgery compared with those living at sea level (29). Another study recently found that compared with low-altitude 


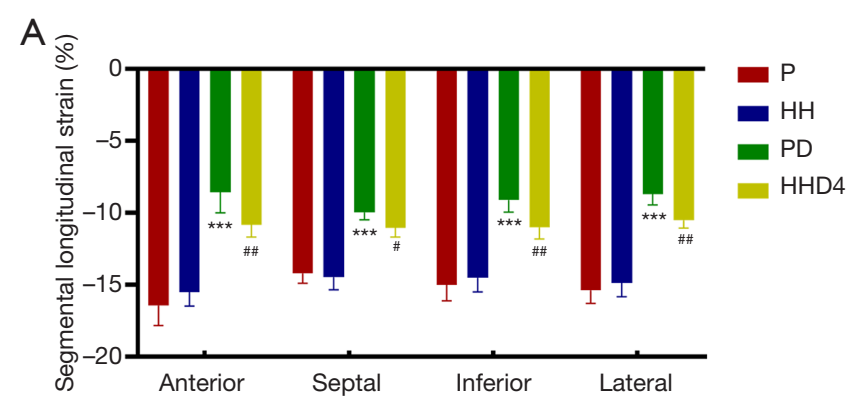

investigated the cardioprotective effect of a natural highaltitude environment on DIC by establishing SpragueDawley rat models at an altitude of $4,250 \mathrm{~m}$. In the current study, we found that exposure to a natural high-altitude environment for 4 weeks effectively improved LV function and strain and reduced myocardial damage and fibrosis from DIC, indicating that a natural high altitude was an effective method for preventing DIC.

CMR has been used to assess multiple manifestations of

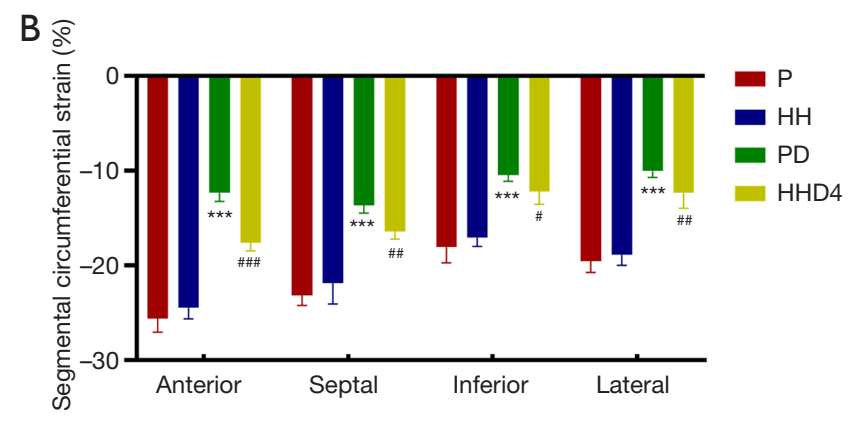
DIC (34). Using serial CMR, Farhad et al. demonstrated that the changes in cardiac structure and function in mice treated with DOX are strongly linked to late mortality (35). MRI-derived strain analysis has also been used to evaluate potential DIC; for example, Ong et al. used myocardial strain imaging with CMR in 41 human epidermal growth factor 2-positive (HER2+) breast cancer patients undergoing chemotherapy. The authors found that there was a significant reduction in LV strain after patients received chemotherapy, which correlated with a subtle decline in LVEF (25). A similar finding was noted in a subsequent study including 125 breast cancer patients treated with epirubicin. Significant reductions in LV longitudinal strain in subendocardial segments were observed after 3 cycles of chemotherapy, while conventional LV systolic and diastolic conventional parameters, as well as LVEF, were relatively spare (36). Other studies have reported that LV regional wall motion abnormalities are closely associated with the development of cancer therapy-related cardiac dysfunction in patients undergoing chemotherapy and may also be an important predictor of DIC (37-41). To the best of our knowledge, we are the first to use strain analysis to evaluate the cardioprotective effect of a natural highaltitude environment on DIC. In this study, we found that LVEF, global strain (GLS, GCS, and GRS) and segmental strain (anterior, septal, inferior, and lateral) decreased in the $\mathrm{PD}$ group compared with the P group and that this decline was inhibited in the HHD4 group. All regional strain parameters, including GLS-b, GLS-m, GLS-a, GCS-b, GCS-m, GCS-a, GRS-b, GRS-m, and GRS-a, showed significant differences between the PD and P groups. All segments except for the basal segments recovered in the HHD4 group, indicating that strain dysfunction in the $\mathrm{LV}$ basal area is relatively severe and difficult to recover from after 4 weeks of exposure to a chronic hypobaric and hypoxic environment, which may be related to the higher mechanical stress at the base of the heart during ventricular filling (42-44). In addition, there was a significant correlation for different forms of global strain with LVEF 


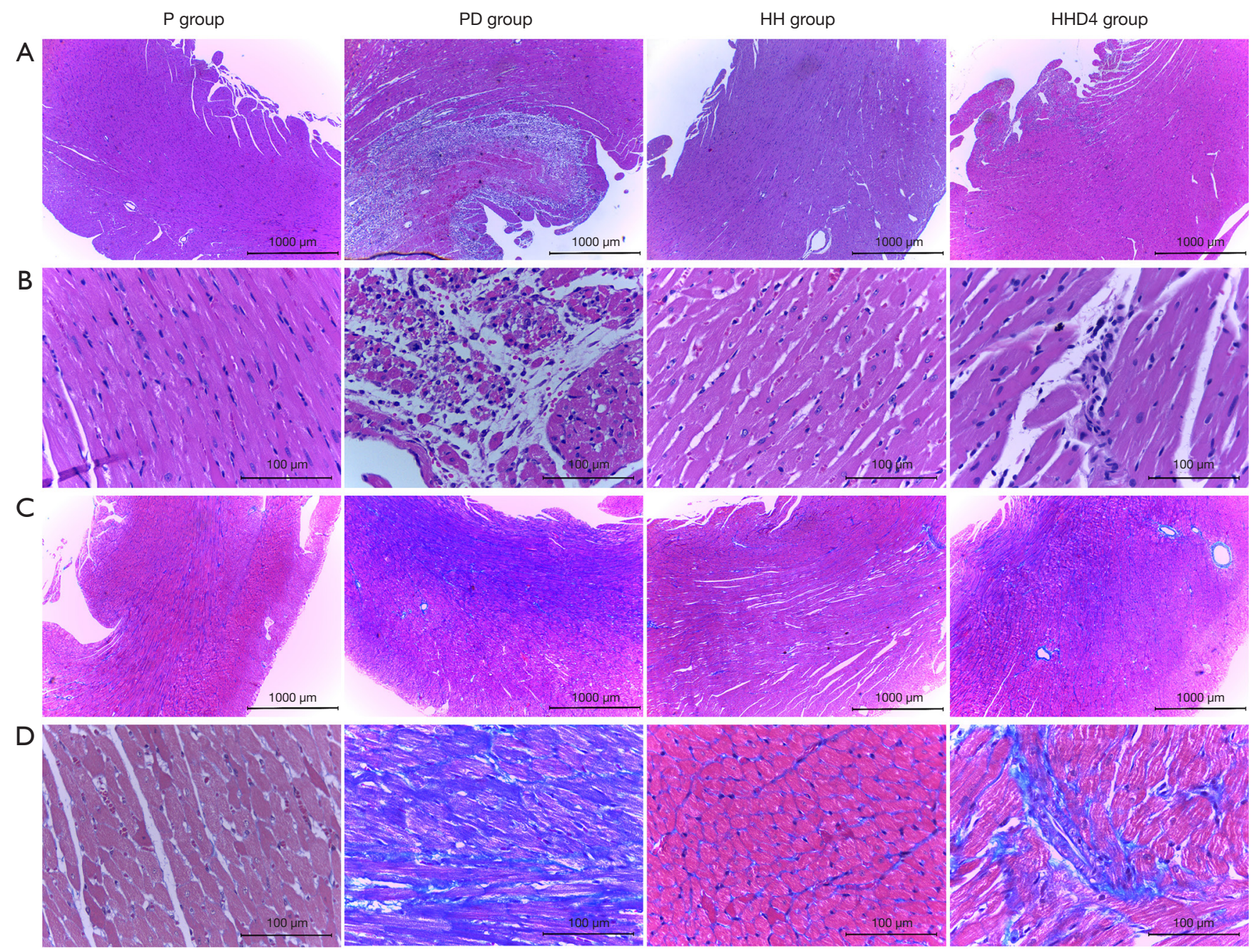

Figure 4 Myocardial tissue sections in the four groups. (A,B) HE staining showing that the extent of myocardial injury was reduced in the HHD4 group. (C,D) MT staining showing that the extent of cardiac fibrosis was reduced in the HHD4 group. HE, hematoxylin-eosin; MT, Masson's trichrome; P, plain; PD, plain + doxorubicin; HH, hypobaric hypoxia; HHD4, hypobaric hypoxia + doxorubicin for 4 weeks.

Table $4 \mathrm{LV}$ strain parameters detected at weeks 4,8 and 12

\begin{tabular}{lccccc}
\hline Parameters & P group & PD group & HHD4 group & HHD8 group & HHD12 group \\
\hline LVEF (\%) & $65.82 \pm 4.24$ & $53.26 \pm 7.16^{\star \star \star}$ & $57.64 \pm 3.16^{\#}$ & $61.26 \pm 4.13^{\# \# \#}$ & $62.07 \pm 3.69^{\# \# \#}$ \\
GLS (\%) & $-16.15 \pm 2.07$ & $-9.83 \pm 1.89^{\star \star \star}$ & $-11.74 \pm 1.42^{\#}$ & $-13.9 \pm 1.78^{\# \# \& \&}$ & $-14.02 \pm 2.48^{\# \# \# \&}$ \\
GCS (\%) & $-21.05 \pm 3.48$ & $-13.62 \pm 4.26^{\star \star \star}$ & $-16.58 \pm 1.68^{\#}$ & $-19.78 \pm 1.69^{\# \# \#}$ & $-19.64 \pm 1.78^{\# \# \# \&}$ \\
GRS (\%) & $45.28 \pm 11.71$ & $23.55 \pm 7.56^{\star \star \star *}$ & $29.31 \pm 6.7^{\# \#}$ & $36.54 \pm 6.11^{\# \# \&}$ & $37.96 \pm 4.06^{\# \# \#}$ \\
\hline
\end{tabular}

The results are presented as the mean $\pm \mathrm{SD}$. ${ }^{\star \star *} \mathrm{P}<0.001$ compared with the $\mathrm{P}$ group; ${ }^{\#} \mathrm{P}<0.05$, ${ }^{\# \#} \mathrm{P}<0.01,{ }^{\# \# \#} \mathrm{P}<0.001$ compared with the PD group; ${ }^{8} \mathrm{P}<0.05$ compared with the HHD4 group. LV, left ventricular; P, plain; PD, plain + doxorubicin; HH, hypobaric hypoxia; HHD4, hypobaric hypoxia + doxorubicin for 4 weeks; HHD8, hypobaric hypoxia + doxorubicin for 8 weeks; HHD12, hypobaric hypoxia + doxorubicin for 12 weeks; LVEF, left ventricular ejection fraction; GLS, global longitudinal strain; GCS, global circumferential strain; GRS, global radial strain. 

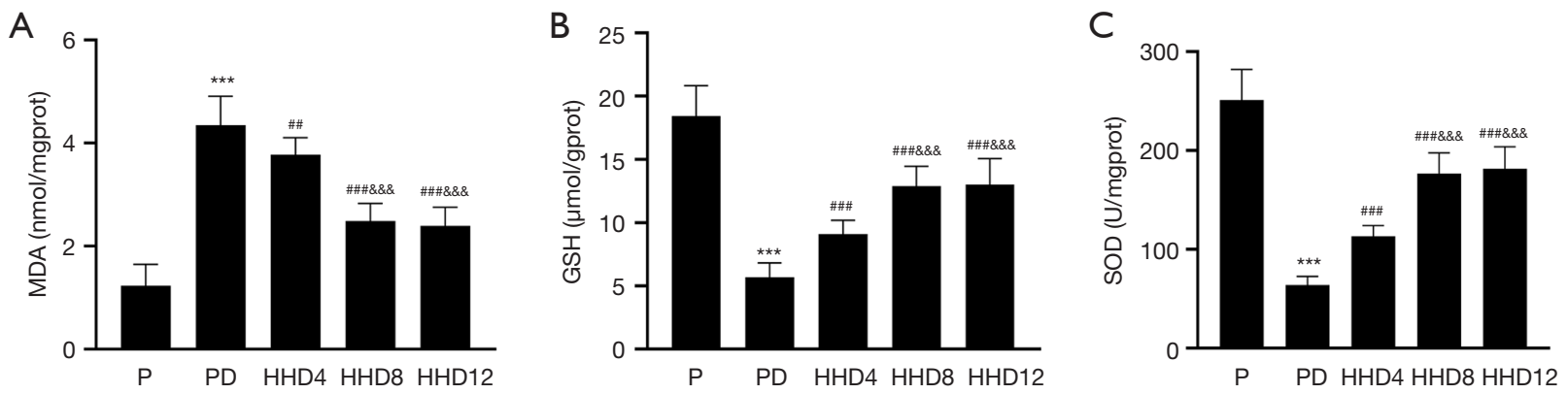

Figure 5 Increasing the total duration of exposure to a high-altitude environment reduced oxidative stress in the heart. (A) MDA levels, (B) GSH levels, and (C) SOD activity were measured in the cardiac tissue of the rats in each group, $n=8$. The data are presented as the mean $\pm \mathrm{SD} .{ }^{* * *} \mathrm{P}<0.001$ compared with the $\mathrm{P}$ group; ${ }^{\# \#} \mathrm{P}<0.01,{ }^{\# \# \#} \mathrm{P}<0.001$ compared with the $\mathrm{PD}$ group; ${ }^{\text {\&\&\& }} \mathrm{P}<0.001$ compared with the HHD4 group. MDA, malondialdehyde; GSH, glutathione; SOD, superoxide dismutase; P, plain; PD, plain + doxorubicin; HHD4, hypobaric hypoxia + doxorubicin for 4 weeks; HHD8, hypobaric hypoxia + doxorubicin for 8 weeks; HHD12, hypobaric hypoxia + doxorubicin for 12 weeks.

Table 5 Intra- and interobserver reliability of measurements for LVEF and segmental strain parameters

\begin{tabular}{lcc}
\hline \multirow{2}{*}{ Parameters } & \multicolumn{2}{c}{ ICC (95\% Cl) } \\
\cline { 2 - 3 } & Intraobserver & Interobserver \\
\hline LVEF (\%) & $0.976(0.954-0.987)$ & $0.914(0.844-0.954)$ \\
GLS (\%) & $0.973(0.950-0.986)$ & $0.897(0.814-0.944)$ \\
GCS (\%) & $0.960(0.926-0.979)$ & $0.924(0.860-0.959)$ \\
GRS (\%) & $0.987(0.976-0.993)$ & $0.954(0.915-0.975)$ \\
GLS-b (\%) & $0.952(0.911-0.974)$ & $0.898(0.815-0.944)$ \\
GLS-m (\%) & $0.950(0.908-0.973)$ & $0.899(0.817-0.945)$ \\
GLS-a (\%) & $0.926(0.865-0.960)$ & $0.851(0.736-0.918)$ \\
GCS-b (\%) & $0.985(0.972-0.992)$ & $0.905(0.827-0.948)$ \\
GCS-m (\%) & $0.953(0.914-0.975)$ & $0.888(0.799-0.939)$ \\
GCS-a (\%) & $0.923(0.860-0.959)$ & $0.836(0.710-0.910)$ \\
GRS-b (\%) & $0.985(0.973-0.992)$ & $0.957(0.921-0.977)$ \\
GRS-m (\%) & $0.987(0.975-0.993)$ & $0.938(0.887-0.967)$ \\
GRS-a (\%) & $0.978(0.959-0.988)$ & $0.931(0.874-0.963)$ \\
\hline
\end{tabular}

LVEF, left ventricular ejection fraction; ICC, intra- and interclass coefficient; $\mathrm{Cl}$, confidence interval; GLS, global longitudinal strain; GCS, global circumferential strain; GRS, global radial strain; GLS-b, basal segment GLS; GLS-m, mid-segment GLS; GLS-a, apical segment GLS; GCS-b, basal segment GCS; GCS-m, mid-segment GCS; GCS-a, apical segment GCS; GRS-b, basal segment GRS; GRS-m, mid-segment GRS; GRS-a, apical segment GRS. and histological fibrosis. These findings suggested that DIC could be partially prevented by chronic hypobaric hypoxia and that the addition of a natural high-altitude environment was an effective method for preventing DIC.

After confirming the validity of exposure to hypobaric hypoxia, we investigated the effect of different durations of this environment. We observed that compared with the HHD4 group, global strain (GLS, GCS, and GRS) increased in the HHD8 and HHD12 groups, while LVEF was unaffected, suggesting that increasing the total time of hypobaric hypoxia exposure further improved cardiac function in DIC. However, LVEF, GLS, GCS, and GRS were not significantly different in the HHD8 and HHD12 groups. This finding might have been due to the reduction in reactive oxygen species production caused by adaptive chronic hypoxia in DIC rats (45). Papandreou et al. were the first to demonstrate that a long-term hypoxic environment downregulates mitochondrial oxygen consumption, which may be responsible for the reduction of oxidative stress (46). Additionally, accumulating evidence suggests that hypobaric hypoxia at high altitude can prevent high levels of oxidative stress and quicken adaptation to a high-altitude environment by increasing the activity of antioxidant enzymes, such as SOD and GSH, in animals (47-49) and in humans (49-52). Although there is no consensus regarding the mechanism of adaptive oxidative stress reduction in rats, in the oxidation system, antioxidant activity is the first line of defense against free radical damage, which can 

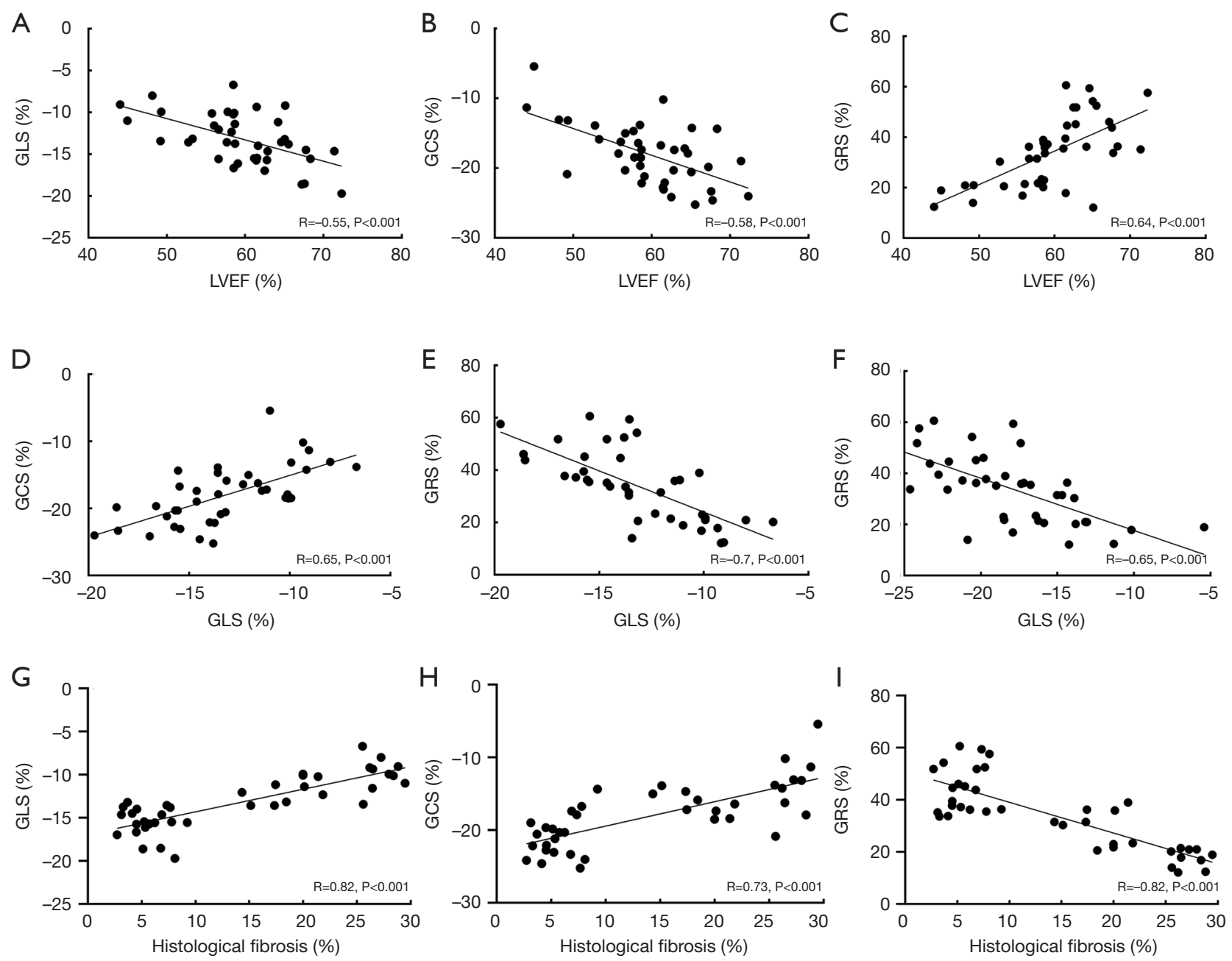

Figure 6 Correlation between LVEF, GLS, GCS, GRS, and histological fibrosis. There was a correlation between LVEF and GLS (A), GCS (B), and GRS (C); a correlation between GLS and GCS (D), GLS and GRS (E), and GCS and GRS (F); and a correlation between myocardial fibrosis and GLS (G), GCS (H), and GRS (I). LVEF, left ventricular ejection fraction; GLS, global longitudinal strain; GCS, global circumferential strain; GRS, global radial strain; R, correlation coefficient.

be disturbed under hypobaric hypoxia-induced oxidative stress (50). Therefore, we focused on indicators of oxidative stress in the different groups and found that compared with that in the $\mathrm{P}$ group, the oxidative stress index in the PD group was significantly increased; however, after exposure to a high-altitude environment, this index was significantly decreased in the HHD4 group and further decreased in the HHD8 and HHD12 groups. These results indicated that adaptation to chronic hypoxia in DIC rats at high altitude for 8 and 12 weeks further alleviated the oxidative stress injury occurring in the heart tissue under hypoxia, which possibly improved the protective effect of chronic hypobaric hypoxia itself on DIC by enhancing the activity of antioxidant enzymes.

\section{Limitations}

The advantages of CMR-TT are its increased stability, short postprocessing time, and semiautomation; however, this technique is susceptible to motion artefacts, poor image quality, and a lack of standardization. In this study, we assessed myocardial deformation by CMR-TT but did not include CMR tagging or a strain-encoded imaging method. CMR tagging is considered the gold standard for assessing 
myocardial deformation (16,53), the use of which is limited by low spatial resolution, tag fading, and complicated postprocessing. Similarly, poor clinical and research experience limited the use of strain-encoded imaging.

\section{Conclusions}

A chronic hypobaric hypoxic environment at high altitude partially prevented cardiac dysfunction; and increased global and segmental strains of the apical, mid-ventricular, anterior, septal, inferior, and lateral segments in DIC rat models; thereby alleviating myocardial injury and fibrosis. In addition, by increasing the total exposure time to chronic hypobaric hypoxia, global strain was further increased, which was likely due to reduced oxidative stress.

\section{Acknowledgments}

Funding: This study was supported by the National Natural Science Foundation of China (no. 81930046, 81771800, and 81829003), the State Key Project of Research and Development Plan of China (no. 2016YFA0201402), and the International Cooperation Project of Science and Technology Plan of Sichuan (no. 2017HH0045).

\section{Footnote}

Conflicts of Interest: All authors have completed the ICMJE uniform disclosure form (available at https://dx.doi. org/10.21037/qims-21-360). The authors have no conflicts of interest to declare.

Ethical Statement: The authors are accountable for all aspects of the work in ensuring that questions related to the accuracy or integrity of any part of the work are appropriately investigated and resolved. All procedures were performed under an approved protocol by the Institutional Animal Care and Use Committee of the Animal Experimental Center, West China Hospital, Sichuan University. The rats were maintained in accordance with the requirements of the National Institutes of Health Guide for the Care and Use of Laboratory Animals and the Association for Assessment and Accreditation of Laboratory Animal Care. All experimental protocols were reviewed and approved by the Experimental Animal Ethics Committee of West China Hospital, Sichuan University, China.

Open Access Statement: This is an Open Access article distributed in accordance with the Creative Commons Attribution-NonCommercial-NoDerivs 4.0 International License (CC BY-NC-ND 4.0), which permits the noncommercial replication and distribution of the article with the strict proviso that no changes or edits are made and the original work is properly cited (including links to both the formal publication through the relevant DOI and the license). See: https://creativecommons.org/licenses/by-nc-nd/4.0/.

\section{References}

1. Minotti G, Menna P, Salvatorelli E, Cairo G, Gianni L. Anthracyclines: molecular advances and pharmacologic developments in antitumor activity and cardiotoxicity. Pharmacol Rev 2004;56:185-229.

2. Hardaway BW. Adriamycin-associated cardiomyopathy: where are we now? updates in pathophysiology, dose recommendations, prognosis, and outcomes. Curr Opin Cardiol 2019;34:289-95.

3. Meinardi MT, van Veldhuisen DJ, Gietema JA, Dolsma WV, Boomsma F, van den Berg MP, Volkers C, Haaksma J, de Vries EG, Sleijfer DT, van der Graaf WT. Prospective evaluation of early cardiac damage induced by epirubicincontaining adjuvant chemotherapy and locoregional radiotherapy in breast cancer patients. J Clin Oncol 2001;19:2746-53.

4. Jiang Q, Li H, Huang X, Yu L, Lueck S, Hu S. Postnatal exposure to hypobaric hypoxia and its impact on inflammation and injury indexes after a cardiac valve procedure. Interact Cardiovasc Thorac Surg 2020;31:789-95.

5. Gu S, Hua H, Guo X, Jia Z, Zhang Y, Maslov LN, Zhang $\mathrm{X}, \mathrm{Ma} \mathrm{H}$. PGC-1 $\alpha$ participates in the protective effect of chronic intermittent hypobaric hypoxia on cardiomyocytes. Cell Physiol Biochem 2018;50:1891-902.

6. Maulik A, Davidson SM, Piotrowska I, Walker M, Yellon DM. Ischaemic preconditioning protects cardiomyocytes from anthracycline-induced toxicity via the PI3K pathway. Cardiovasc Drugs Ther 2018;32:245-53.

7. Galán-Arriola C, Villena-Gutiérrez R, Higuero-Verdejo MI, Díaz-Rengifo IA, Pizarro G, López GJ, MolinaIracheta A, Pérez-Martínez C, García RD, González-Calle D, Lobo M, Sánchez PL, Oliver E, Córdoba R, Fuster V, Sánchez-González J, Ibanez B. Remote ischaemic preconditioning ameliorates anthracycline-induced cardiotoxicity and preserves mitochondrial integrity. Cardiovasc Res 2021;117:1132-43.

8. Chang JC, Lien CF, Lee WS, Chang HR, Hsu YC, Luo 
YP, Jeng JR, Hsieh JC, Yang KT. Intermittent hypoxia prevents myocardial mitochondrial $\mathrm{Ca} 2+$ overload and cell death during ischemia/reperfusion: the role of reactive oxygen species. Cells 2019;8:564.

9. Gangwar A, Paul S, Ahmad Y, Bhargava K. Intermittent hypoxia modulates redox homeostasis, lipid metabolism associated inflammatory processes and redox posttranslational modifications: Benefits at high altitude. Sci Rep 2020;10:7899.

10. Aguilar M, González-Candia A, Rodríguez J, CarrascoPozo C, Cañas D, García-Herrera C, Herrera EA, Castillo RL. Mechanisms of cardiovascular protection associated with intermittent hypobaric hypoxia exposure in a rat model: role of oxidative stress. Int J Mol Sci 2018;19:366.

11. Huang XS, Chen HP, Yu HH, Yan YF, Liao ZP, Huang QR. Nrf2-dependent upregulation of antioxidative enzymes: a novel pathway for hypoxic preconditioningmediated delayed cardioprotection. Mol Cell Biochem 2014;385:33-41.

12. Nguyen T, Nioi P, Pickett CB. The Nrf2-antioxidant response element signaling pathway and its activation by oxidative stress. J Biol Chem 2009;284:13291-5.

13. Ostadal B, Kolar F. Cardiac adaptation to chronic highaltitude hypoxia: beneficial and adverse effects. Respir Physiol Neurobiol 2007;158:224-36.

14. Zhou ZN, Zhuang JG, Wu XF, Zhang Y, Cherdrungsi P. Tibetans retained innate ability resistance to acute hypoxia after long period of residing at sea level. J Physiol Sci 2008;58:167-72.

15. Ammirati E, Moroni F, Sormani P, Peritore A, Milazzo A, Quattrocchi G, Cipriani M, Oliva F, Giannattasio C, Frigerio M, Roghi A, Camici PG, Pedrotti P. Quantitative changes in late gadolinium enhancement at cardiac magnetic resonance in the early phase of acute myocarditis. Int J Cardiol 2017;231:216-21.

16. Scatteia A, Baritussio A, Bucciarelli-Ducci C. Strain imaging using cardiac magnetic resonance. Heart Fail Rev 2017;22:465-76.

17. Truong UT, Li X, Broberg CS, Houle H, Schaal M, Ashraf M, Kilner P, Sheehan FH, Sable CA, Ge S, Sahn DJ. Significance of mechanical alterations in single ventricle patients on twisting and circumferential strain as determined by analysis of strain from gradient cine magnetic resonance imaging sequences. Am J Cardiol 2010;105:1465-9.

18. Kovács Z, Kormányos Á, Domsik P, Kalapos A, Lengyel C, Ambrus N, Ajtay Z, Piros GÁ, Forster T, Nemes A. Left ventricular longitudinal strain is associated with mitral annular fractional area change in healthy subjectsResults from the three-dimensional speckle tracking echocardiographic MAGYAR-Healthy Study. Quant Imaging Med Surg 2019;9:304-11.

19. Kostakou PM, Kostopoulos VS, Tryfou ES, Giannaris VD, Rodis IE, Olympios CD, Kouris NT. Subclinical left ventricular dysfunction and correlation with regional strain analysis in myocarditis with normal ejection fraction. A new diagnostic criterion. Int J Cardiol 2018;259:116-21.

20. Zhu J, Chen Y, Xu Z, Wang S, Wang L, Liu X, Gao F. Non-invasive assessment of early and acute myocarditis in a rat model using cardiac magnetic resonance tissue tracking analysis of myocardial strain. Quant Imaging Med Surg 2020;10:2157-67.

21. Liu T, Wang C, Li S, Zhao Y, Li P. Age- and genderrelated normal references of right ventricular strain values by tissue tracking cardiac magnetic resonance: results from a Chinese population. Quant Imaging Med Surg 2019;9:1441-50.

22. Hu LW, Liu XR, Wang Q, Barton GP, Ouyang RZ, Sun AM, Guo C, Han TT, Yao XF, François CJ, Zhong YM. Systemic ventricular strain and torsion are predictive of elevated serum NT-proBNP in Fontan patients: a magnetic resonance study. Quant Imaging Med Surg 2020;10:485-95.

23. Oikonomou EK, Kokkinidis DG, Kampaktsis PN, Amir EA, Marwick TH, Gupta D, Thavendiranathan P. Assessment of prognostic value of left ventricular global longitudinal strain for early prediction of chemotherapyinduced cardiotoxicity: a systematic review and metaanalysis. JAMA Cardiol 2019;4:1007-18.

24. Chakouri N, Farah C, Matecki S, Amedro P, Vincenti M, Saumet L, Vergely L, Sirvent N, Lacampagne A, Cazorla O. Screening for in-vivo regional contractile defaults to predict the delayed Doxorubicin Cardiotoxicity in Juvenile Rat. Theranostics 2020;10:8130-42.

25. Ong G, Brezden-Masley C, Dhir V, Deva DP, Chan KKW, Chow CM, Thavendiranathan D, Haq R, Barfett JJ, Petrella TM, Connelly KA, Yan AT. Myocardial strain imaging by cardiac magnetic resonance for detection of subclinical myocardial dysfunction in breast cancer patients receiving trastuzumab and chemotherapy. Int J Cardiol 2018;261:228-33

26. Arciniegas Calle MC, Sandhu NP, Xia H, Cha SS, Pellikka PA, Ye Z, Herrmann J, Villarraga HR. Two-dimensional speckle tracking echocardiography predicts early subclinical cardiotoxicity associated with anthracyclinetrastuzumab chemotherapy in patients with breast cancer. 
BMC Cancer 2018;18:1037.

27. Fridrik MA, Jaeger U, Petzer A, Willenbacher W, Keil F, Lang A, Andel J, Burgstaller S, Krieger O, Oberaigner W, Sihorsch K, Greil R. Cardiotoxicity with rituximab, cyclophosphamide, non-pegylated liposomal doxorubicin, vincristine and prednisolone compared to rituximab, cyclophosphamide, doxorubicin, vincristine, and prednisolone in frontline treatment of patients with diffuse large B-cell lymphoma: a randomised phase-III study from the Austrian Cancer Drug Therapy Working Group Arbeitsgemeinschaft Medikamentöse Tumortherapie AGMT(NHL-14). Eur J Cancer 2016;58:112-21.

28. Faeh D, Gutzwiller F, Bopp M; Swiss National Cohort Study Group. Lower mortality from coronary heart disease and stroke at higher altitudes in Switzerland. Circulation 2009;120:495-501.

29. Hu Y, Sun Q, Li Z, Chen J, Shen C, Song Y, Zhong Q. High basal level of autophagy in high-altitude residents attenuates myocardial ischemia-reperfusion injury. J Thorac Cardiovasc Surg 2014;148:1674-80.

30. Lyu Q, Bai Y, Cheng J, Liu H, Li S, Yang J, Wang Z, Ma Y, Jiang M, Dong D, Yan Y, Shi Q, Ren X, Ma J. Intermittent short-duration reoxygenation protects against simulated high altitude-induced pulmonary hypertension in rats. FASEB J 2021;35:e21212.

31. Yang Z, Sun H, Su S, Nan X, Li K, Jin X, Jin G, Li Z, Lu D. Tsantan Sumtang restored right ventricular function in chronic hypoxia-induced pulmonary hypertension rats. Front Pharmacol 2020;11:607384.

32. Li N, Chen K, Bai J, Geng Z, Tang Y, Hou Y, Fan F, Ai X, Hu Y, Meng X, Wang X, Zhang Y. Tibetan medicine Duoxuekang ameliorates hypobaric hypoxia-induced brain injury in mice by restoration of cerebrovascular function. J Ethnopharmacol 2021;270:113629.

33. Asemu G, Papousek F, Ostádal B, Kolár F. Adaptation to high altitude hypoxia protects the rat heart against ischemia-induced arrhythmias. Involvement of mitochondrial K(ATP) channel. J Mol Cell Cardiol 1999;31:1821-31.

34. Galán-Arriola C, Lobo M, Vílchez-Tschischke JP, López GJ, de Molina-Iracheta A, Pérez-Martínez C, Agüero J, Fernández-Jiménez R, Martín-García A, Oliver E, Villena-Gutierrez R, Pizarro G, Sánchez PL, Fuster V, Sánchez-González J, Ibanez B. Serial magnetic resonance imaging to identify early stages of anthracycline-induced cardiotoxicity. J Am Coll Cardiol 2019;73:779-91.

35. Farhad H, Staziaki PV, Addison D, Coelho-Filho OR, Shah RV, Mitchell RN, Szilveszter B, Abbasi SA,
Kwong RY, Scherrer-Crosbie M, Hoffmann U, JeroschHerold M, Neilan TG. Characterization of the changes in cardiac structure and function in mice treated with anthracyclines using serial cardiac magnetic resonance imaging. Circ Cardiovasc Imaging 2016. doi: 10.1161/ CIRCIMAGING.115.003584.

36. Chang WT, Feng YH, Kuo YH, Chen WY, Wu HC, Huang CT, Huang TL, Chen ZC. Layer-specific distribution of myocardial deformation from anthracyclineinduced cardiotoxicity in patients with breast cancer-From bedside to bench. Int J Cardiol 2020;311:64-70.

37. de Barros MVL, Macedo AVS, Sarvari SI, Faleiros MH, Felipe PT, Silva JLP, Edvardsen T. Left ventricular regional wall motion abnormality is a strong predictor of cardiotoxicity in breast cancer patients undergoing chemotherapy. Arq Bras Cardiol 2019;112:50-6.

38. Akam-Venkata J, Kadiu G, Galas J, Lipshultz SE, Aggarwal $\mathrm{S}$. Left ventricle segmental function in childhood cancer survivors using speckle-tracking echocardiography. Cardiol Young 2019;29:1494-500.

39. Keramida K, Farmakis D, Bingcang J, Sulemane S, Sutherland S, Bingcang RA, Ramachandran K, Tzavara C, Charalampopoulos G, Filippiadis D, Kouris N, Nihoyannopoulos P. Longitudinal changes of right ventricular deformation mechanics during trastuzumab therapy in breast cancer patients. Eur J Heart Fail 2019;21:529-35.

40. Li H, Liu C, Zhang G, Wang C, Sun P, Du G, Tian J. The early alteration of left ventricular strain and dyssynchrony index in breast cancer patients undergoing anthracycline therapy using layer-specific strain analysis. Echocardiography 2019;36:1675-81.

41. Saijo Y, Kusunose K, Okushi Y, Yamada H, Toba H, Sata M. Relationship between regional left ventricular dysfunction and cancer-therapy-related cardiac dysfunction. Heart 2020;106:1752-8.

42. Sengupta PP, Tajik AJ, Chandrasekaran K, Khandheria BK. Twist mechanics of the left ventricle: principles and application. JACC Cardiovasc Imaging 2008;1:366-76.

43. Modesto K, Sengupta PP. Myocardial mechanics in cardiomyopathies. Prog Cardiovasc Dis 2014;57:111-24.

44. Cheung YF, Hong WJ, Chan GC, Wong SJ, Ha SY. Left ventricular myocardial deformation and mechanical dyssynchrony in children with normal ventricular shortening fraction after anthracycline therapy. Heart 2010;96:1137-41.

45. Ali SS, Hsiao M, Zhao HW, Dugan LL, Haddad GG, Zhou D. Hypoxia-adaptation involves mitochondrial 
metabolic depression and decreased ROS leakage. PLoS One 2012;7:e36801.

46. Papandreou I, Cairns RA, Fontana L, Lim AL, Denko NC. HIF-1 mediates adaptation to hypoxia by actively downregulating mitochondrial oxygen consumption. Cell Metab 2006;3:187-97.

47. Zhu M, Xu M, Zhang K, Li J, Ma H, Xia G, Li X, Zhang $\mathrm{B}$, Shi H. Effect of acute exposure to hypobaric hypoxia on learning and memory in adult Sprague-Dawley rats. Behav Brain Res 2019;367:82-90.

48. Jochmans-Lemoine A, Revollo S, Villalpando G, Valverde I, Gonzales M, Laouafa S, Soliz J, Joseph V. Divergent mitochondrial antioxidant activities and lung alveolar architecture in the lungs of rats and mice at high altitude. Front Physiol 2018;9:311.

49. Sharma S, Singh Y, Sandhir R, Singh S, Ganju L, Kumar B, Varshney R. Mitochondrial DNA mutations contribute to high altitude pulmonary edema via increased

Cite this article as: Wan Y, Zhu D, He B, Guo Y, Wang L, Dingda D, Laji A, Wang C, Zhang Y, Gao F. Protective effect of a chronic hypobaric hypoxic environment at high altitude on cardiotoxicity induced by doxorubicin in rats: a $7 \mathrm{~T}$ magnetic resonance study. Quant Imaging Med Surg 2022;12(1):711-725. doi: 10.21037/qims-21-360 oxidative stress and metabolic reprogramming during hypobaric hypoxia. Biochim Biophys Acta Bioenerg 2021;1862:148431.

50. Tang XG, Wen J, Zhang XS, Jiang DC. Association between decreased osteopontin and acute mountain sickness upon rapid ascent to $3500 \mathrm{~m}$ among young Chinese men. J Travel Med 2018. doi: 10.1093/jtm/tay075.

51. Vij AG, Dutta R, Satija NK. Acclimatization to oxidative stress at high altitude. High Alt Med Biol 2005;6:301-10.

52. Janocha AJ, Comhair SAA, Basnyat B, Neupane M, Gebremedhin A, Khan A, Ricci KS, Zhang R, Erzurum SC, Beall CM. Antioxidant defense and oxidative damage vary widely among high-altitude residents. Am J Hum Biol 2017. doi: 10.1002/ajhb.23039.

53. Pedrizzetti G, Claus P, Kilner PJ, Nagel E. Principles of cardiovascular magnetic resonance feature tracking and echocardiographic speckle tracking for informed clinical use. J Cardiovasc Magn Reson 2016;18:51. 